\title{
AVALIAÇÃO DOS INDICADORES EM SAÚDE DAS CRIANÇAS ACOMPANHADAS PELA PASTORAL DA CRIANÇA
}

\author{
EVALUATION OF HEALTH INDICATORS IN CHILDREN ACCOMPANIED BY THE MINISTRY OF CHILD
}

Tainara Lôrena dos Santos Ferreira, Laiana Carla Pereira Gomes, Daísy Vieira de Araújo, Fábia Barbosa de Andrade

\section{RESUMO}

Objetivo: Este estudo objetivou avaliar o perfil de atendimento das crianças acompanhadas pela Pastoral da Criança da cidade de Lajes Pintadas, estado do Rio Grande do Norte. Métodos: Trata-se de um estudo exploratório-descritivo, de cunho transversal e de abordagem quantitativa. Os dados foram coletados no período de junho a agosto de 2013, com uma amostra de 53 crianças menores de cinco anos, cadastradas na Pastoral da Criança. Resultados: Observou-se que $100 \%$ das crianças estão com as vacinas atualizadas; enquanto $79,3 \%$ apontam que as crianças sempre comparecem à consulta de puericultura. Conclusão: Conclui-se que a Pastoral da Criança contribui para que as crianças acompanhadas tenham mais qualidade de vida e saúde, considerando que colaboram para a prevenção de doenças incentivando às consultas de puericultura, além da procura por serviços de saúde.

Descritores: Saúde da Criança. Saúde Pública. Rede Social. Avaliação em Saúde.

\section{ABSTRACT}

Objective: The objective of this study was to evaluate the child care profile of the children of Pastoral da Criança in the city of Lajes Pintadas, state of Rio Grande do Norte. Methods: This is an exploratory-descriptive, cross-sectional and quantitative approach. Data were collected from June to August 2013, with a sample of 53 children under the age of five enrolled in the Pastoral da Criança. Results: It was observed that $100 \%$ of the children have the vaccines updated; while $79.3 \%$ indicated that children always attended the child care consultation. Conclusion: It is concluded that Pastoral of the Child (Pastoral da Criança - in Portuguese) contributes to the children's quality of life and health, considering that they collaborate in the prevention of diseases by encouraging child-care consultations, as well as the search for health services.

Descriptors: Child Health. Public Health. Social Networking. Health Evaluation.
Autor Principal

Nome: Tainara Lôrena dos Santos Ferreira Formação Profissional: Acadêmica do Curso de Enfermagem da UFRN/FACISA

Filiação Institucional: Universidade Federal do Rio Grande do Norte (UFRN) / Faculdade de Ciências da Saúde do Trairi (FACISA)

Nome: Laiana Carla Pereira Gomes Formação Profissional: Enfermeira Filiação Institucional: Universidade Federal do Rio Grande do Norte (UFRN) / Faculdade de Ciências da Saúde do Trairi (FACISA)

\section{Nome: Daisy Vieira de Araújo} Formação Profissional: Enfermeira. Mestre em Enfermagem

Filiação Institucional: Universidade Federal do Rio Grande do Norte (UFRN) / Faculdade de Ciências da Saúde do Trairi (FACISA)

Nome: Fábia Barbosa de Andrade Formação Profissional: Enfermeira. Mestre em Enfermagem. Doutora em Ciências da Saúde pela Universidade Federal do Rio Grande do Norte (UFRN). Filiação Institucional: Universidade Federal do Rio Grande do Norte (UFRN) / Faculdade de Ciências da Saúde do Trairi (FACISA) 


\section{INTRODUÇÃO}

O acompanhamento do crescimento e desenvolvimento da saúde da criança é de grande valor para uma vida futura com boa qualidade, principalmente, daquelas que vivem em vulnerabilidade social, como são as crianças cadastradas pela Pastoral da Criança. Destarte o papel da Atenção Primária em Saúde (APS) através das ações de promoção, proteção e recuperação da saúde para a população infantil a partir das consultas de puericultura.

Na perspectiva de saúde como completo bem estar, emerge a necessidade de acompanhamento de famílias que se encontram em vulnerabilidade social, a fim de possibilitar a criação do elo entre as ações de saúde e a comunidade. Embora se saiba que a partir da APS, bem como no que versa o Sistema Único de Saúde (SUS) houve grandes avanços de saúde, no que tange a cobertura dos serviços, acesso e resolutividade, ainda há muitos desafios postos, dentre eles, possibilitar as famílias em risco à saúde e vulnerabilidade social uma melhor qualidade de vida e saúde a partir da educação em saúde, ações de promoção da saúde e prevenção de agravos.

No âmbito da saúde da criança torna-se importante que a puericultura tenha início na primeira semana de vida da criança, o mais próximo possível da alta hospitalar, pois é nesse período que a mãe e a família precisam de muita ajuda e orientações sobre o recém-nascido, bem como se faz necessária a garantia do acesso dos recém-nascidos e suas mães, usuárias da comunidade, ao atendimento de enfermagem, médico, vacinação, amamentação, teste do pezinho, bem como a continuidade destes seguimentos e a consulta de puerpério'.

Tendo em vista a puericultura ser uma atividade de prevenção, devendo a criança ser atendida por uma equipe de profissionais e esse atendimento ser realizado por meio de consultas de rotina, é necessário que se tenham informações a respeito das condições de saúde, moradia, saneamento básico, escolaridade e economia familiar, cujas crianças são atendidas para que as ações de saúde dispensadas sejam efetivas².

A APS deve ser eficiente na realização do acompanhamento do crescimento e desenvolvimento das crianças, principalmente daquelas que se apresentam em vulnerabilidade social, com o objetivo de manter uma ligação eficaz entre a criança, a família e o serviço de saúde. Desta maneira, o contato da criança com os serviços de saúde é tido como uma oportunidade integrada e uma ação resolutiva, de promoção da saúde e caráter educativo das equipes, fato que torna o acompanhamento sistemático do crescimento e desenvolvimento da criança o eixo central do atendimento.

Como nos serviços de saúde, há o programa de acompanhamento de Crescimento e Desenvolvimento na Pastoral da Criança da Igreja Católica, uma organização não-governamental de ação social da Conferência Nacional dos Bispos do Brasil (CNBB), que tem se destacado nacional e internacionalmente por se propor a ajudar na redução dos agravos aos quais estão acometidas as crianças brasileiras pobres. Por meio da organização de ações básicas de saúde, nutrição e educação, atua também na promoção da saúde de gestantes.

Concernente a pastoral da criança destaca-se que esta possui caráter voluntário, atuando na promoção humana, via combate à mortalidade infantil, à violência doméstica e à marginalidade social. Visando ofertar suporte às famílias $\mathrm{e}$ às comunidades, os membros da pastoral são capacitados quando a alimentação saudável, imunização, e as doenças que surgem na infância, acompanhando mensalmente gestantes e crianças de zero a seis anos de idade 3 .

Uma importante característica distintiva dos movimentos sociais é a sua capacidade de gerar identidades coletivas. A construção de identidades é essencial para que haja uma ligação entre os membros que se identificam enquanto participantes de um grupo, que dividem valores e objetivos, e mostram um comprometimento com a causa do movimento social. Assim, a identidade coletiva proporcionada pela participação em movimentos sociais ajuda no 
processo de ação coletiva, propicia incentivos pessoais aos seus integrantes e reforça o comprometimento individual ${ }^{4}$.

Neste contexto, por entender a importância de possibilitar uma melhor qualidade de vida às crianças, grupo considerado como área prioritária do cuidado, e por saber ainda que as crianças cadastradas na Pastoral da Criança da Igreja Católica apresentam-se como uma população vulnerável, o referido estudo se propõe a avaliar o perfil de atendimento das crianças assistidas e acompanhadas pela Pastoral da Criança.

\section{Metodologia}

Trata-se de um estudo exploratório-descritivo, de cunho transversal e abordagem quantitativa, realizado no município de Lajes Pintadas, Rio Grande do Norte, Brasil. A cidade compreende 130,211 Km² de área territorial e possui aproximadamente 4.612 habitantes. É considerada uma região pobre e pouco desenvolvida, com IDH em torno de 0,655 e PIB per capita de $\mathrm{R} \$ 4.810,66^{5}$.

O presente estudo trata-se de um recorte da pesquisa intitulada Perfil do crescimento e desenvolvimento nas crianças acompanhadas pela pastoral da criança: um estudo de avaliação em saúde. A população estudada é constituída por 62 crianças cadastradas e acompanhadas pela Pastoral da Criança do município supracitado.

A amostra foi composta apenas por 53 crianças de ambos os sexos que se encontravam com 4 anos, 11 meses e 29 dias até o dia da entrevista, que estavam cadastradas na Pastoral da Criança há mais de seis meses e cujos responsáveis assinaram o Termo de Consentimento Livre e Esclarecido (TCLE); 09 crianças da população deste estudo não participaram por não se encontrarem em sua residência familiar ou terem mudado de endereço.

As entrevistas ocorreram nas próprias residências dos responsáveis pelas crianças participantes da Pastoral da Criança, em dias da semana, de segunda-feira a sexta-feira, com o acompanhamento dos líderes responsáveis por cada família, onde foram realizadas visitas domiciliares para obtenção das respostas do formulário, para melhor alcance dos dados coletados.

Os dados foram coletados no período de junho ao início de agosto de 2013, através de um formulário estruturado composto por questões construídas com base nas diretrizes e no manual norteador do que preconiza a Pastoral da Criança, sendo este um guia e caderno do líder de acompanhamento nos seguintes aspectos: vacinação, peso, crescimento e desenvolvimento, diarréia, aleitamento materno exclusivo e atendimento das crianças nos serviços de saúde.

Para análise dos dados, foi utilizada a estatística descritiva por meio do conhecimento de médias, medianas, desvio padrão, frequências absolutas e relativas, bem como o software Stata 9.0 , sendo posteriormente os dados apresentados em tabelas e discutidos a partir da estatística descritiva e da literatura pertinente

Concernente aos aspectos éticos, o estudo seguiu todas as recomendações constantes da Resolução 466/2012 do Conselho Nacional de Saúde, referentes à pesquisa com seres humanos, sendo aprovado pelo Comitê de Ética em Pesquisa da Faculdade de Ciências da Saúde do Trairi - Universidade Federal do Rio Grande do Norte, sob parecer de número 311.618.

\section{Resultados}

Os resultados revelam, quanto à idade em meses das crianças da pastoral da criança, que a média corresponde a 31,19; mediana a 32,0 e desvio padrão a 14,8. Já a idade mínima foi de 6 meses e máxima de 59 meses. Com relação 
ao peso das crianças, a média obtida em quilogramas foi de 13,98Kg; mediana de 13,0 Kg e desvio padrão de 3,746Kg, com valor mínimo de $8,0 \mathrm{Kg}$ e máximo de $25 \mathrm{Kg}$. A média da estatura foi $90,48 \mathrm{~cm}$; mediana de $92,0 \mathrm{~cm}$, enquanto 0 desvio padrão foi de 12,63cm e, assim, a estatura mínima entre as crianças foi de $66 \mathrm{~cm}$ e a máxima de $110 \mathrm{~cm}$. A amostra foi constituída por $56,6 \%$ ( $n=30)$ crianças do sexo feminino e $43,4 \%(n=23)$ do sexo masculino.

Tabela 1 - Classificação da qualidade dos serviços prestados pela pastoral da criança na ótica dos usuários. Lajes Pintadas/RN, Brasil, 2014.

\begin{tabular}{lllll}
\hline Variáveis & Ótimo & Bom & Regular & Total \\
\hline Atualização das vacinas & $86,8 \%(n=46)$ & $\begin{array}{l}13,2 \% \\
(n=07)\end{array}$ & 00 & $100 \%$ \\
Acompanhamento e ganho de & $62,3 \%(n=33)$ & $\begin{array}{l}37,7 \% \\
(n=20)\end{array}$ & 00 & $(n=53)$ \\
peso & & $37,7 \%$ & & $100 \%$ \\
& & 00 & $(n=53)$ \\
Representatividade da pastoral & $62,3 \%(n=33)$ & $100 \%$ \\
Serviço de saúde - consulta de & & $(n=20)$ & & $(n=53)$ \\
puericultura & $54,7 \%(n=29)$ & $\begin{array}{l}35,9 \% \\
(n=19)\end{array}$ & $1,9 \%$ & $92,5 \%$ \\
& & $49 \%$ & $3,8 \%$ & $\left(n=49^{*}\right)$ \\
Crescimento e desenvolvimento & $47,2 \%(n=25)$ & $(n=26)$ & $(n=02)$ & $100 \%$ \\
& & & & $(n=53)$ \\
\hline
\end{tabular}

*A diferença da amostra está relacionada aos casos que não se aplicaram

Na tabela 1, observa-se a classificação da qualidade dos serviços prestados pela pastoral da criança na ótica de usuários. $E$, no que diz respeito à atualização das vacinas prestadas pela pastoral, $86,8 \%(n=46)$ classificaram como ótimo, seguido por 13,2\% ( $n=07)$ que classificaram como bom. Quanto à qualidade do acompanhamento prestado pela pastoral e ganho de peso, 62,3\% ( $n=33)$ consideraram como ótimo e 37,7\% $(n=20)$ afirmaram ser bom. No tocante ao questionamento de como as mães julgam a representatividade da Pastoral da Criança para os direitos à saúde da criança na cidade, $62,3 \%(n=33)$ responderam que é ótimo. No que diz respeito ao acompanhamento da consulta de puericultura para a criança, 54,7\% (29) das mães disseram que é ótimo. Em relação ao crescimento e desenvolvimento das crianças, 49\% (26) afirmaram ser bom. Quanto ao trabalho da Pastoral da Criança diante do incentivo ao AME, 47,15\% (25) apontaram como ótimo, seguido por $47,15 \%$ (25) que afirmaram ser bom. 
Tabela 2 - Distribuição das crianças da pastoral com as vacinas atualizadas. Lajes Pintadas/RN, Brasil, 2014.

\begin{tabular}{ccccc}
\hline Variáveis & Sempre & Às vezes & Nunca & Total \\
\hline Vacinas atualizadas & $100 \%$ & 00 & 00 & $100 \%$ \\
& $(n=53)$ & & & $(n=53)$ \\
Aprazamento das vacinas & $98,1 \%$ & $1,9 \%$ & 00 & $100 \%$ \\
Orientação dos líderes & $(n=52)$ & $(n=01)$ & & $(n=53)$ \\
Visitas da pastoral/ vacinas & $98,1 \%$ & 00 & $1,9 \%$ & $100 \%$ \\
atualizadas & $(n=52)$ & & $(n=01)$ & $(n=53)$ \\
& $92,5 \%$ & $7,5 \%$ & 00 & $100 \%$ \\
& $(n=49)$ & $(n=04)$ & & $(n=53)$ \\
\hline
\end{tabular}

A tabela 2, sobre a atualização das vacinas das crianças da pastoral da criança, aponta que $100 \%$ ( $n=53$ ) das crianças sempre estão com as vacinas atualizadas. Mostra também, que 98,1\% ( $n=52)$ referiram que o serviço de saúde sempre realiza o aprazamento para que as vacinas não atrasem e $98,1 \%(n=52)$ relataram que, sempre que tem vacina atrasada, os líderes da pastoral orientam os pais a levarem a criança ao serviço de saúde para receber as doses que faltam. No que se refere ainda às visitas da Pastoral da Criança, realizadas mensalmente, ajudar a manter o calendário vacinal atualizado, 92,5\% ( $n=49)$ mencionaram que sempre.

Tabela 3 - Distribuição do peso das crianças da pastoral da criança. Lajes Pintadas/RN, Brasil, 2014.

\begin{tabular}{cccccc}
\hline Variáveis & Sempre & Às vezes & Raramente & Nunca & Total \\
\hline Incentivo da pastoral para & $94,3 \%$ & $3,8 \%$ & $1,9 \%$ & 00 & $100 \%$ \\
alimentação saudável & $(\mathrm{n}=50)$ & $(\mathrm{n}=02)$ & $(\mathrm{n}=01)$ & & $(\mathrm{n}=53)$ \\
Contribuição da pastoral para o & $90,6 \%$ & $7,5 \%$ & $1,9 \%$ & & $100 \%$ \\
peso adequado & $(\mathrm{n}=48)$ & $(\mathrm{n}=04)$ & $(\mathrm{n}=01)$ & 00 & $(\mathrm{n}=53)$ \\
Informe sobre o peso da & $81,1 \%$ & $3,8 \%$ & 00 & $15,1 \%$ & $100 \%$ \\
criança mensalmente & $(\mathrm{n}=43)$ & $(\mathrm{n}=02)$ & & $(\mathrm{n}=08)$ & $(\mathrm{n}=53)$ \\
Peso adequado das crianças & $62,3 \%$ & $35,8 \%$ & $1,9 \%$ & & $100 \%$ \\
para as mães & $(\mathrm{n}=33)$ & $(\mathrm{n}=19)$ & $(\mathrm{n}=01)$ & 00 & $(\mathrm{n}=53)$ \\
\hline
\end{tabular}

$\mathrm{Na}$ tabela 3, com relação ao incentivo da pastoral para que as crianças atendidas tenham uma alimentação saudável, 94,3\% (n=50) relataram que isso sempre acontece. Quanto à contribuição da pastoral para o peso adequado, $90,6 \%(n=48)$ apontaram que a pastoral sempre contribui para que as crianças tenham um peso adequado. No que concerne às mães serem informadas mensalmente sobre o peso da criança, 81,1\% ( $n=43)$ afirmaram que sempre, enquanto $15,1 \%$ ( $n=08$ ) afirmaram nunca acontecer. $E$, quanto às mães considerarem adequado o peso das crianças que a elas é referido, $62,3 \%(n=33)$ afirmaram sempre considerar adequado, seguido por $35,8 \%(n=19)$ que consideram às vezes. 
Tabela 4 - Distribuição do atendimento das crianças da pastoral da criança nos serviços de saúde. Lajes Pintadas/RN, Brasil, 2014.

\begin{tabular}{cccccc}
\hline Variáveis & Sempre & Às vezes & Raramente & Nunca & Total \\
\hline Criança que comparecem à & $79,3 \%$ & $5,7 \%$ & $7,5 \%$ & $7,5 \%$ & $100 \%$ \\
consulta de puericultura & $(n=42)$ & $(n=03)$ & $(n=04)$ & $(n=04)$ & $(n=53)$ \\
Crianças atendidas nos & $71,7 \%$ & $26,4 \%$ & $1,9 \%$ & & $100 \%$ \\
serviços de saúde & $(n=38)$ & $(n=14)$ & $(n=01)$ & 00 & $(n=53)$ \\
Crianças bem atendidas nos & $60,4 \%$ & $30,2 \%$ & $7,5 \%$ & $1,9 \%$ & $100 \%$ \\
serviços de saúde & $(n=32)$ & $(n=16)$ & $(n=04)$ & $(n=01)$ & $(n=53)$ \\
\hline
\end{tabular}

A tabela 4, que diz respeito ao atendimento das crianças da pastoral da criança nos serviços de saúde, indica que $79,3 \%$ (n=42) sempre comparecem à consulta de puericultura no serviço de saúde local, de acordo com o dia marcado. Quanto ao atendimento nos serviços de saúde, $71,7 \%$ ( $n=38$ ) referiram sempre, seguido por $26,4 \%$ ( $n=14$ ) que afirmaram às vezes. Em relação a serem bem atendidas nos serviços de saúde, 60,4\% ( $n=32)$ disseram que sempre são bem atendidas no serviço de saúde, enquanto $30,2 \%$ (n=16) afirmaram que às vezes.

\section{Discussão}

Um serviço de qualidade é dever de todos os prestadores, para que, assim, se tenha uma melhor qualidade de saúde e de vida da população. A assistência oferecida deve ser avaliada constantemente, com o intuito de aperfeiçoar cada vez mais os serviços oferecidos e buscar analisá-los, para que haja um melhor planejamento das intervenções necessárias e, desta maneira, beneficiar todos os usuários da assistência.

No âmbito de serviços de saúde da criança, portanto, busca-se a qualidade da atenção à saúde, a efetividade da assistência das ações e serviços prestados a esta população e o fortalecimento da proposta de vigilância em saúde da criança, que trata-se de uma diretriz operacional com o objetivo de reduzir os indicadores de morbimortalidade infantil, possibilitando assim o alcance de metas propostas a nível nacional e internacional, em busca de melhores resultados da saúde infantil 6 .

O acompanhamento regular do crescimento e do desenvolvimento infantil é de grande importância, pois monitora e beneficia as condições de saúde, assim como de nutrição da criança assistida. Os índices antropométricos são utilizados como o principal critério desse acompanhamento. Esse indicativo baseia-se no conhecimento de que a discrepância entre as necessidades fisiológicas e a ingestão de alimentos causam alterações físicas nos indivíduos, desde o sobrepeso e a obesidade, até graves quadros de desnutrição ${ }^{7}$

Avaliar e, consequentemente, melhorar a qualidade dos cuidados de saúde oferecidos aos cidadãos, além de assegurar a todos os utilizadores acesso a cuidados de qualidade, em tempo útil e com custos adequados, é o grande desafio para os profissionais da área da saúde. Como consequência, verifica-se que a avaliação da qualidade dos cuidados de saúde tem despertado, nos últimos tempos, interesse crescente. Que tem se manifestado não só ao nível dos decisores políticos e das administrações, mas também no seio dos profissionais de saúde e dos próprios usuários ${ }^{8}$.

A importância que as vacinas têm na proteção à saúde e na prevenção de doenças imunopreveníveis é 
inquestionável, sobretudo durante a infância. Como consequência, as autoridades de saúde, em todo o mundo, instituíram calendários específicos de vacinas, de acordo com a faixa etária infantil. No Brasil, como em outros países, o Ministério da Saúde desenvolve programas de imunização e promove campanhas periodicamente, com o intuito de controlar e erradicar doenças, a partir da vacinação maciça de crianças $^{9}$.

Este estudo mostra que a pastoral da criança contribui para que as crianças atendidas mantenham as vacinas atualizadas (Tabela 2), fator de total importância no controle de saúde das crianças, uma vez que colabora para a prevenção e eliminação de doenças imunopreveníveis e aumenta a cobertura vacinal do município. Ainda, mensalmente, são realizadas as visitas domiciliares em que os líderes orientam os pais para que a criança seja levada ao serviço de saúde, onde os pais ou responsáveis têm a oportunidade de manter atualizada a caderneta de vacinação de suas crianças.

A procura de uma faixa de peso saudável e o combate ao excesso de peso e obesidade deve ser uma inquietação de todos os profissionais que trabalham com as crianças e suas famílias. Todas as oportunidades são boas para corrigir hábitos alimentares desequilibrados, promover a atividade física e combater o sedentarismo. Regra geral, trata-se de um problema familiar e, como tal, a abordagem de um elemento poderá beneficiar todos os membros da família ${ }^{10}$.

Ao promover o controle do peso, o ritmo metabólico pode ser favorecido pelo fracionamento das refeições. Por isso, recomenda-se o consumo de quatro a seis refeições por dia (desjejum, merenda, almoço, lanche, jantar e ceia), além da orientação para que o consumo de grandes volumes de alimentos em uma única refeição e intervalos prolongados entre as refeições sejam evitados. A substituição de refeições como o almoço e o jantar por lanches não é aconselhada, haja vista que não contempla diversos nutrientes. Em alguns casos, o lanche pode ser mais calórico e, por conter pouca fibra, sua digestão é mais rápida, o que diminui o tempo de sensação de saciedade ${ }^{11}$.

Diante do exposto (Tabela 3), de maneira geral, a pastoral da criança traz muitas contribuições para uma vida saudável das crianças, tendo em vista que contribui para indicar dietas e, consequentemente, ganho de peso adequado. Porém, a pastoral pode contribuir ainda mais para prevenir a desnutrição e a obesidade, ou até mesmo, combatê-las através da implantação das hortas caseiras, do incentivo à alimentação saudável, de atividade física, ou ainda com mensuração de medidas antropométricas que visem o alcance de grandes benefícios futuros.

A terminologia de crescimento e desenvolvimento corresponde a fenômenos distintos, embora correlacionados. Crescimento significa aumento do corpo, como um todo ou em algumas de suas partes, e pode ser mensurado em centímetros ou gramas. Constitui o aumento do tamanho das células. Desenvolvimento denota capacidade do indivíduo em realizar funções cada vez mais complexas, ou seja, desenvolver controle neuromuscular, destreza e funções que só podem ser mensuradas por meio de provas ou testes funcionais ${ }^{12}$.

O crescimento e o desenvolvimento das crianças (Tabela 4) também dependem da idade gestacional e do peso ao nascer, pois estes podem trazer riscos biológicos para o desenvolvimento da criança. Os líderes da pastoral podem auxiliar ainda mais as famílias acompanhadas durante as visitas e as reuniões de reflexão, ao mostrar qual o crescimento e desenvolvimento certo para cada criança.

A Atenção à Saúde da Criança representa um marco, pois ao propor o atendimento à saúde infantil no contexto do cuidado integralizado e a sua adoção, mostra-se como um passo importante para o reconhecimento dos direitos da criança. São condições que convergem para a integralidade do cuidado e compreendem o desenvolvimento das ações de prevenção e assistência a agravos como objetivos que, além da redução da mortalidade infantil, apontam para 0 
compromisso de se prover qualidade de vida para a criança. Portanto, resume-se, no desafio de possibilitar à criança crescer e desenvolver-se com todo o seu potencial ${ }^{13}$.

Um atendimento digno e de qualidade é direito de todos os cidadãos e usuários de saúde. Desta maneira, as mães devem levar seus filhos à consulta de puericultura, para realizar um acompanhamento de rotina, que priorize os cuidados com a saúde da criança e busque, assim, reduzir os problemas de morbidade e mortalidade, além de proporcionar uma maior qualidade de vida da população infantil.

\section{Conclusão}

A Pastoral da Criança contribui para que as crianças acompanhadas possuam mais qualidade de vida e saúde, tendo em vista colaborar para a prevenção de doenças, através do incentivo à vacinação e orientação das mães pelos líderes, sobre os indicadores de oportunidades e conquistas do desenvolvimento das crianças, com relação à nutrição, saúde e educação, serviços estes que condizem com o que há preconizado pelo Ministério da Saúde.

Sabe-se que a saúde da criança é vista como área prioritária do cuidado e entende-se que há a importância de uma assistência integral, efetiva e humanizada, que compreenda o usuário em seu contexto biopsicossocial. Assim, os resultados deste estudo mostraram a contribuição da Pastoral da Criança no estabelecimento do acompanhamento das crianças na Atenção Primária em Saúde. Ainda existem fragilidades e desafios postos para prestar uma melhor assistência, mas percebeu-se avanços e a colaboração da Pastoral da Criança para a transformação social através da educação em saúde, na busca pelos direitos humanos.

Ressalta-se que além da Pastoral da Criança, existem outras instituições, programas e políticas públicas, que contribuem para a promoção, melhores condições de saúde e redução da mortalidade infantil como, por exemplo: Conselho Municipal dos Direitos da Criança e do Adolescente (CMDCA), Conselhos tutelares, Bolsa Família e o SUS. Espera-se, portanto, que este estudo venha fortalecer a contribuição de outras instituições e programas para promover a saúde da população infantil e direcionar reformulações de ações e serviços de saúde, com vistas a uma melhor qualidade de vida, bem como uma assistência em saúde efetiva e contínua.

\section{Referências}

1. Brasil. Programa de Atenção à Saúde da Criança e do Adolescente Protocolo de Enfermagem. Prefeitura municipal de Ribeirão Preto. Ribeirão Preto: Secretária de saúde, 2010.

2. Fadul ALF. Desenvolvimento e crescimento de crianças de zero a cinco anos: proposta de um protocolo de puericultura para o município de Ibirité. Trabalho de conclusão do curso de especialização em Atenção Básica em Saúde da Família, Universidade Federal de Minas Gerais. Corinto $\square$ MG, 2012.

3. Teotônio RV, Sousa LSN, Lima LHO, Oliveira EAR, Figueiredo IGA, Cruz TMGA. Perfil antropométrico e epidemiológico das crianças acompanhadas pelos líderes da pastoral da criança. Revista de Enfermagem da UFPI. 2015; 4(4): 41-8.

4. Frutuoso JRA, Maia JL. A Pastoral da Criança enquanto movimento social: uma análise de redes e identidades. Revista Habitus: revista eletrônica dos alunos de graduação em Ciências Sociais - IFCS/UFRJ, Rio de Janeiro. 2009 Jul; 7(1): 45-59. Disponível em: <www.habitus.ifcs.ufrj.br>. Acesso em: 01 de dez. de 2012.

5. Instituto Brasileiro de Geografia e Estatística (IBGE), 2010. Rio de Janeiro: IBGE. Disponível em: <http://www. ibge.gov.br/cidadesat/xtras/perfil.php?codmun=240680\&r=> Acessado em: 26 de março de 2013. 
6. Ferreira TLS, Bezerra HS, Melo JTA, Nobre TTX, Andrade FB. Evaluation of Child Health by Basic Indicators of Health: Historical Series 2005-2014. Health. 2018; 10:289-99.

7. Brasil. Ministério da saúde. Secretaria de Atenção à Saúde. Departamento de Atenção Básica. CoordenaçãoGeral da Política de Alimentação e Nutrição Vigilância alimentar e nutricional - SISVAN. Série A. Normas e Manuais Técnicos. Brasília: Ministério da saúde, 2004.

8. Souza et al. Avaliação da qualidade em saúde: a importância do ajustamento pelo risco na análise de resultados na doença coronária. Revista portuguesa de saúde pública. 2008; Volume temático: 7.

9. Silveira ASA et al. Controle de vacinação de crianças matriculadas em escolas municipais da cidade de São Paulo. Rev Esc Enferm USP. São Paulo, SP. 2007; 2(41): 299-305.

10. Brasil. Ministério da saúde. Direção geral da saúde. Circular normativa. Consultas de Vigilância de Saúde Infantil e Juvenil: atualização das curvas de crescimento. №: 05/DSMIA. Brasília: Ministério da saúde, 2006.

11. Brasil. Ministério da saúde. Secretaria de Atenção à Saúde. Departamento de Atenção Básica. Obesidade: Cadernos de Atenção Básica - n. ${ }^{0}$ 12. Série A. Normas e Manuais Técnicos. $1^{a}$ ed. Brasília: Ministério da saúde, 2006.

12. Carabolante AC, Ferriani MGC. O crescimento e desenvolvimento de crianças na faixa etária de 12 a 48 meses em creche na periferia da cidade de Ribeirão Preto - SP. Revista Eletrônica de Enfermagem. Ribeirão Preto, SP. 2003; $5(1): 28-34$.

13. Erdmann AL, Sousa FGM. Cuidando da criança na Atenção Básica de Saúde: atitudes dos profissionais da saúde. O Mundo da Saúde São Paulo. 2(33):150-160, São Paulo, 2009. 